\title{
The method of particular solutions for the Helmholtz equation
}

\author{
G. E. Sneddon* W. W. Read* \\ (Received 29 October 2004, 8 revised April 2005)
}

\begin{abstract}
We have previously showed that the advection-diffusion equation in steady hill-slope seepage problems can be reduced to the solution of the Helmholtz equation in two dimensions. Initially, solutions were found using an analytic series method (or the method of particular solutions). However, the accuracy of these solutions is limited by ill-conditioning in the set of basis functions as the number of basis functions increases. Here we show that these problems are overcome by choosing a different set of basis functions and modifying the method of particular solutions as suggested by Betcke and Trefethen. The different methods are tested on a number of simple domains. In most cases spectral convergence is obtained for the eigenvalue.
\end{abstract}

${ }^{*}$ School of Mathematical and Physical Sciences, James Cook University, Townsville, Australia. mailto: graeme.sneddon@jcu.edu.au

See http://anziamj.austms.org.au/V46/CTAC2004/Sned for this article, (c) Austral. Mathematical Soc. 2005. Published June 29, 2005. ISSN 1446-8735 


\section{Contents}

1 Introduction

C545

2 Analytic series method

C546

3 The modified method

C548

4 Comparison of methods

C550

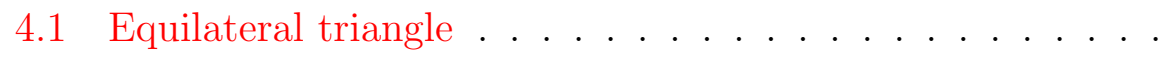

C551

4.2 Semicircle

C553

4.3 Parabolic boundaries

C556

5 Conclusion

C556

References

C557

\section{Introduction}

The Method of Particular Solutions (MPS) has been used to solve partial differential equations such as the Helmholtz equation $\Delta \phi=-\lambda \phi$ on a domain $P$ with $\phi=0$ on $\partial P$. Perhaps the best known application of this method is that of Fox, Henrici and Moler [1]: they applied the method to the L-shaped domain, and the result features in the well-known MATLAB logo.

Our interest comes from seeking solutions to the advection-diffusion equation describing contaminant transport in hill-slope seepage problems on an irregular domain. Read, Sneddon and Bode [2] showed that under certain conditions, the solution of this equation can be reduced to finding solutions of the Helmholtz equation on the domain. In that paper we used an "Analytic Series Method" to solve the equation. This was effectively the MPS.

Betcke and Trefethen [3] suggested a modified approach to this problem 
(the Modified Method of Particular Solutions or MMPS). In Sections 2 and 3 these approaches are described and different sets of basis functions presented. In Section 4 these different approaches are compared by solving the Helmholtz equation on simple domains such as the equilateral triangle, the semicircle and various parabolic shapes.

\section{Analytic series method}

In [2] we considered the advection-diffusion equation in steady hill-slope seepage problems. We showed that if analytic approximations for the hydraulic head $\Phi(X, Y)$ and the conjugate stream function $\Psi(X, Y)$ are available, and if the magnitude of the stream velocity is approximately constant, then the solution of the equation could be reduced to solving

$$
\nabla^{2} \phi(x, y)+\lambda \phi(x, y)=0,
$$

in transformed coordinates $x$ and $y$.

For a given value of $\lambda$, solutions of equation (1) can be found using separation of variables in either Cartesian or polar coordinates. Given a domain $P$ with boundary $\partial P$, we choose a set of such elementary functions $\left\{v_{k}(x, y)\right\}$ that satisfy (1). We then look for a solution of the form

$$
\phi(x, y)=\sum_{k=1}^{\infty} a_{k} v_{k}(x, y),
$$

which is zero on the boundary $\partial P$. In many cases the $v_{k}(x, y)$ themselves can be chosen to be zero on some parts of the boundary and it remains to choose the $a_{k}$ so that $\phi$ is zero on a single curve $y=f^{t}(x)$.

In practice, we truncate the sum in (2) after $N$ terms and collocate at $N$ 


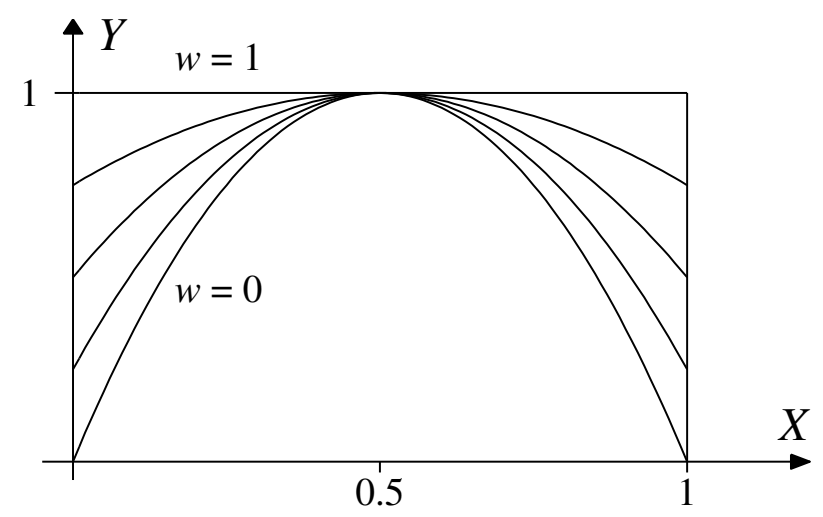

Figure 1: Domains for the five test problems of [2].

points $x_{i}, i=1, \ldots, N$, along this curve. That is

$$
\sum_{k=1}^{N} a_{k} v_{k}^{t}\left(x_{i}\right)=0, \quad \text { for } i=1, \ldots, N,
$$

where $v_{k}^{t}\left(x_{i}\right)=v_{k}\left(x_{i}, f^{t}\left(x_{i}\right)\right)$. In matrix form $V^{t} \boldsymbol{a}=\mathbf{0}$, where $V_{i k}^{t}=v_{k}^{t}\left(x_{i}\right)$ and $(\boldsymbol{a})_{k}=a_{k}$. In general $V^{t}$ will depend on $\lambda$ and must be singular to allow a non-zero solution for $\boldsymbol{a}$. We assume that this can only occur when $\lambda$ is an eigenvalue or natural frequency of the Helmholtz equation (1), and (2) is an eigenfunction.

The method was initially applied to a region contained within the unit square with boundaries $x=0, x=1, y=0$ and

$$
y=w+4(1-w) x(1-x), \quad w=0: 0.25: 1 .
$$

The five domains are shown in Figure 1. In the case $w=1$ the solution that is zero on each of the boundaries is known to be $\phi(x, y)=\sin k \pi x \sin m \pi y$. For this reason we chose the basis functions

$$
v_{k}(x, y)=\sin k \pi x \begin{cases}\sin \gamma_{k} y, & k \leq n_{0}, \\ \sinh \gamma_{k} y / \cosh \gamma_{k}, & k>n_{0}\end{cases}
$$


with $\gamma_{k}^{2}=\left|\lambda-k^{2} \pi^{2}\right|$ and $n_{0}=\lfloor\sqrt{\lambda} / \pi\rfloor$. This choice of basis functions was suitable for values of $w \lesssim 1$. However there were difficulties for values of $w$ close to zero. These difficulties were partly due to the requirement that even in the case $w=0$, the solution (2) will still be zero along the lines $x=0$ and $x=1$, as well as on the upper boundary. The presence of the sinh function for large values of $N$ also meant that the functions themselves were not wellconditioned. For these reasons, a different set of basis functions is adopted for the purpose of the comparisons in Section 4.

The functions used still have the form $\sin c_{k} x \sin d_{k} y$, but instead of $c_{k}$ being required to be $k \pi$ with $k$ an integer, $c_{k}$ and $d_{k}$ are chosen to be the components of a vector with magnitude $\sqrt{\lambda}$. The directions of the vectors $\left(c_{k}, d_{k}\right)$ could be chosen to be evenly distributed between 0 and $\pi / 2$. Thus

$$
c_{k}=\sqrt{\lambda} \cos \theta_{k}, \quad d_{k}=\sqrt{\lambda} \sin \theta_{k}, \quad k=1, \ldots N,
$$

with $\theta_{k}=\pi k /(2 N)$. Initially, we are interested in solutions that are symmetric about $x=0.5$. Therefore, the functions actually chosen are

$$
v_{k}(x, y)=W_{N, k}(x, y)=\cos c_{k}(x-0.5) \sin d_{k} y .
$$

There is one further advantage in choosing the functions (7). The two dimensional Fourier transform, $F(\omega, \sigma)$, of any solution of (1) will be zero except on the curve $\omega^{2}+\sigma^{2}=\lambda$. If $\phi$ is a sum of the terms (7), then the coefficients of the sum will give the value of the transform (apart from a phase factor) along this curve. This can be used as an independent check of the values of these coefficients.

\section{The modified method}

The method described above is essentially the MPs described by Fox et al. [1], the main difference being that they used combinations of Bessel functions and 
sine functions for their basis functions. Recently Betcke and Trefethen [3] drew attention to some of the problems with this method. These problems are related to the ill-conditioning of the $v_{k}$ over the domain and the fact that there will be solutions for $\boldsymbol{a}$ for which $\phi(x, y)$ will be almost zero throughout the domain. Their resolution of this problem was to extend the matrix $V^{t}$ so that it includes values of the basis functions at points in the interior of the domain as well as on the boundary. In addition they evaluated the basis functions at more than $N$ points on the boundary. In their notation, they constructed the matrix

$$
A(\lambda)=\left[\begin{array}{c}
A_{B}(\lambda) \\
A_{I}(\lambda)
\end{array}\right] .
$$

The matrix $A_{B}(\lambda)$ is the same as the matrix $V^{t}$ used previously, except that it will have more rows, corresponding to the larger number of boundary points.

An orthonormal basis of $A(\lambda)$ can be found using an "economy size" $Q R$ decomposition of $A$. Hence

$$
A(\lambda)=\left[\begin{array}{l}
Q_{B}(\lambda) \\
Q_{I}(\lambda)
\end{array}\right] R,
$$

where $R$ is a square upper triangular matrix. The boundary conditions will be satisfied (approximately) if $\lambda$ is chosen so that the smallest singular value of $Q_{B}(\lambda)$ is a minimum. Once $\lambda$ is determined, the coefficients in the expansion (2) are chosen to satisfy $A_{B}(\lambda) \boldsymbol{a}=Q_{B}(\lambda) R \boldsymbol{a} \approx \mathbf{0}$. This will be the case if $R \boldsymbol{a}=\boldsymbol{u}$ where $\boldsymbol{u}$ is the right singular vector of $Q_{B}$ corresponding to the smallest singular value. This approach ensures that the solution for $\phi(x, y)$ is non-zero inside the domain.

Betcke and Trefethen initially applied this method to the L-shaped region of Figure 2. The re-entrant corner at the origin in Figure 2 is singular in the sense that $\alpha=\pi / \vartheta$ is not an integer. Consequently, the solution cannot be analytic there. For this reason the solution was expanded in terms of Bessel functions centred at the origin. The choice

$$
v_{k}(r, \theta)=J_{\alpha k}(\sqrt{\lambda} r) \sin \alpha k \theta
$$




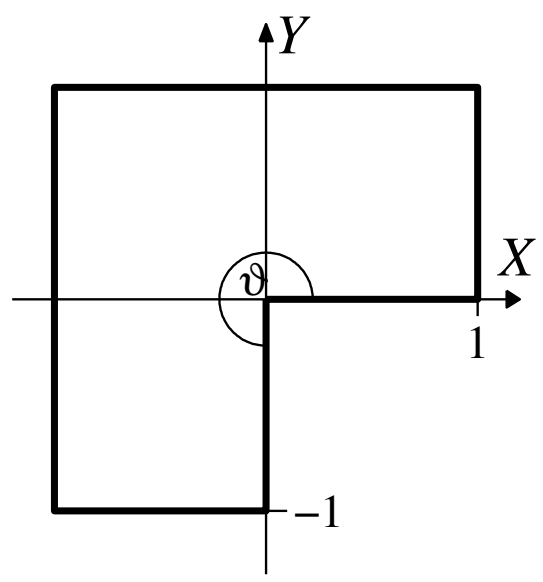

Figure 2: The L-shaped domain.

ensures that each of the basis functions are zero on those parts of the boundary along $x=0$ and $y=0$. The coefficients $\boldsymbol{a}$ and $\lambda$ could be chosen as described above in order that the solution would be approximately zero on the remaining parts of the boundary.

In Section 4 these functions are used to find solutions on simple domains. Since we are interested in the solutions that are symmetric about $x=0.5$, the functions used are

$$
v_{k}(r, \theta)=B_{\alpha, k}(r, \theta)=J_{\alpha k}(\sqrt{\lambda} r) \sin \alpha k \theta+J_{\alpha k}\left(\sqrt{\lambda} r^{\prime \prime}\right) \sin \alpha k \theta^{\prime \prime},
$$

where the meaning of the symbols is shown in Figure 3. The symbols $r^{\prime}$ and $\theta^{\prime}$ are used in Section 4.2

\section{Comparison of methods}

In this section, the different choices of basis functions are each used on some simple domains to check the convergence of the methods with $N$ and to see if 


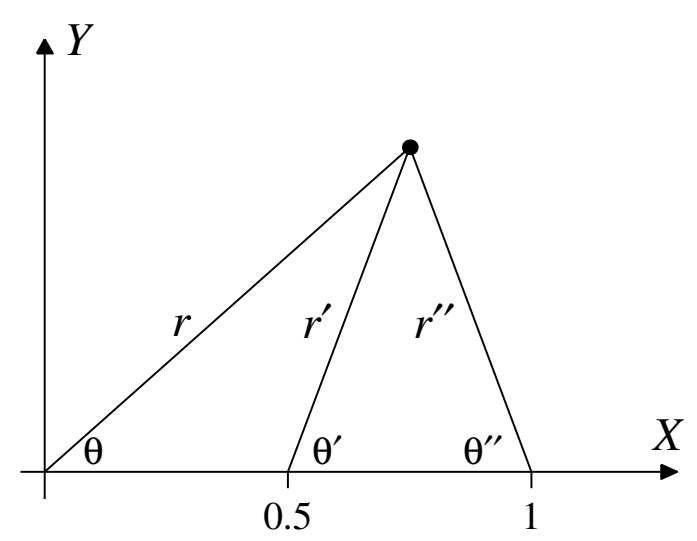

Figure 3: Symbols used to define $B_{\alpha, k}$.

any method is significantly better than the other. The MMPS is used with 20 boundary points and 20 interior points chosen at random. The comparison is made between the sets of basis functions $W_{N, k}$ and $B_{\alpha, k}$ and another set yet to be described.

\subsection{Equilateral triangle}

The method can be applied to the equilateral triangle with vertices at $(0,0)$, $(1,0)$ and $(1 / 2, \sqrt{3} / 2)$. The solution for the fundamental mode is

$$
\phi(\boldsymbol{r})=\sin \sqrt{\lambda} \boldsymbol{k}_{1} \cdot \boldsymbol{r}+\sin \sqrt{\lambda} \boldsymbol{k}_{2} \cdot \boldsymbol{r}+\sin \sqrt{\lambda} \boldsymbol{k}_{3} \cdot \boldsymbol{r},
$$

with $\boldsymbol{k}_{1}=(0,1), \boldsymbol{k}_{2}=-\frac{1}{2}(\sqrt{3}, 1), \boldsymbol{k}_{3}=\frac{1}{2}(\sqrt{3},-1)$ and $\lambda=16 \pi^{2} / 3$ (see [4] for a recent discussion). The errors in the calculated eigenvalue are shown in Figure 4 for both sets of eigenfunctions. In view of the nature of the exact solution, it is no surprise that the wave functions give extremely accurate results when $N$ is a multiple of 3 . The eigenvalue and the solution are obtained to machine precision. 


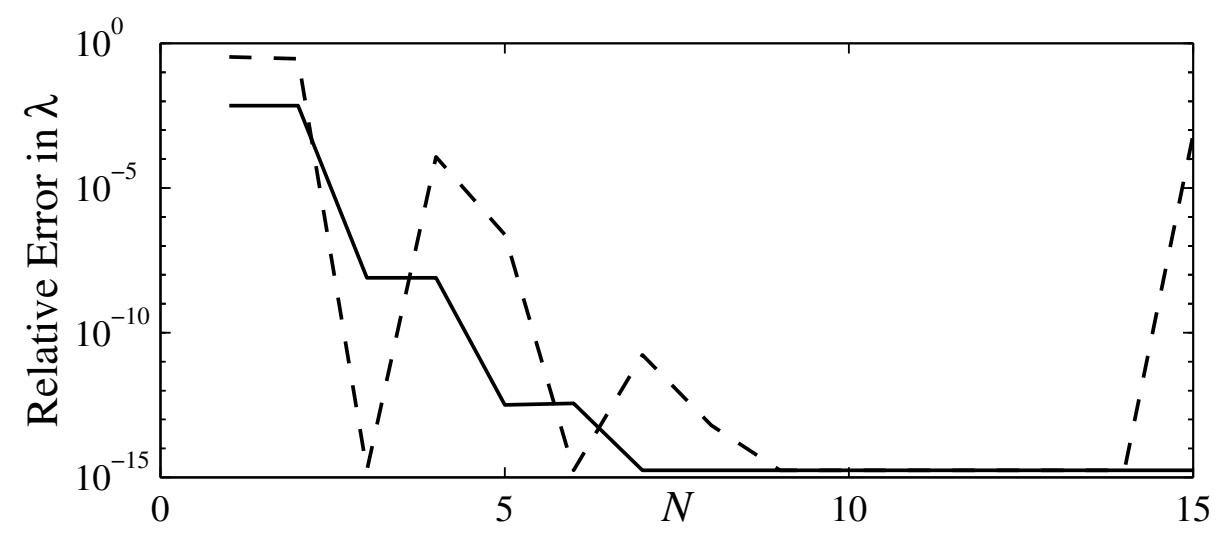

Figure 4: The relative error in $\lambda$ versus $N$ for the triangular domain. The dashed line is for $W_{N, k}$ and the solid line for $B_{\alpha, k}$.

For the expansion in Bessel functions, $\alpha=\pi /(\pi / 3)$ in this case, and so the Bessel functions to be used in the series are $J_{3 k}$. In general, this expansion is more accurate than the plane wave expansion except when $N$ is a multiple of 3. The stepped nature of the graph of the error against $N$ is a surprise initially. Furthermore, the coefficients of the expansion are zero for even values of $k$ and the remainder appear to be equal to each other. This feature can be explained by writing each term in (12) as $\sin (\sqrt{\lambda} r \sin (\theta+2 n \pi / 3))$ and using the identity $\sin (z \sin \theta)=\sum_{k=-\infty}^{\infty} J_{k}(z) \sin (k \theta)$. Equation (12) becomes

$$
\phi(\boldsymbol{r})=\sum_{k=-\infty}^{\infty}(\sin k \theta+\sin k(\theta+2 \pi / 3)+\sin k(\theta+4 \pi / 3)) J_{k}(\sqrt{\lambda} r) .
$$

Terms where $k$ is not a multiple of 3 will vanish. Also, the $k$ th term will either be equal to the $-k$ th term or will cancel with it. Thus

$$
\phi(\boldsymbol{r})=6 \sum_{k=1}^{\infty} \sin 3(2 k-1) \theta J_{3(2 k-1)}(\sqrt{\lambda} r) .
$$




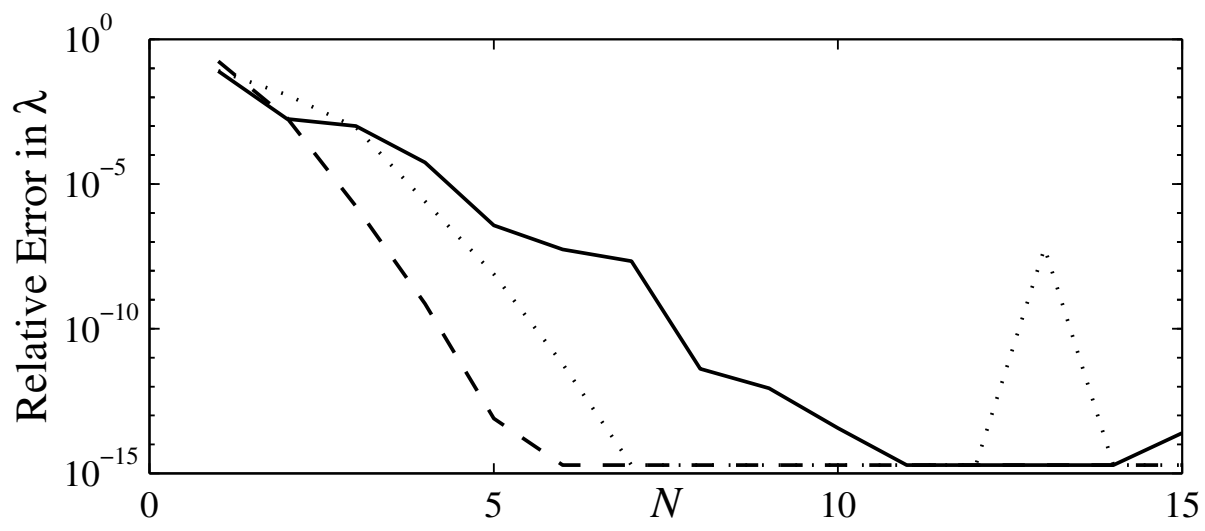

Figure 5: The relative error in $\lambda$ versus $N$ for the semicircular domain. The dashed line is for $W_{N, k}$, the solid line for $B_{\alpha, k}$ and the dotted line for $B_{\alpha, k}^{p}$.

The same result is obtained using the Bessel functions centred at $(1,0)$, thus verifying the result obtained numerically that $\phi(\boldsymbol{r})=3 \sum_{k=1}^{\infty} B_{3,2 k-1}(\boldsymbol{r})$.

\subsection{Semicircle}

For the semicircle with radius $\frac{1}{2}$ and centre $\left(\frac{1}{2}, 0\right)$, the fundamental solution is $\phi(\boldsymbol{r})=J_{1}\left(\sqrt{\lambda} r^{\prime}\right) \sin \theta^{\prime}$, with $\sqrt{\lambda} / 2$ the first zero of $J_{1}$. The Fourier transform of this function (shifted so that the centre is at the origin rather than at $\left(\frac{1}{2}, 0\right)$ ) is zero except on the circle $\omega^{2}+\sigma^{2}=\lambda$, where it is proportional to $\sin \beta$ with $\beta=\arg (\omega+i \sigma)$. The convergence to the eigenvalue using the functions $W_{N, k}$ is shown in Figure 5. For fewer than 12 functions in the expansion, the values of the coefficients $a_{k}$ closely match the values of $\sin (\pi k /(2 N))$. For larger values of $N$, these coefficients showed large variability and huge oscillations, although this did not always affect the calculation of the eigenfunction. This feature has also been noted by Betcke and Trefethen.

Unlike the previous example, the boundary is not composed of straight 
line segments. However, the angle (with the tangent) at the corners is $\pi / 2$ and so we take $\alpha=2$. The final solution itself will not be forced to be zero along these tangent lines $(x=0$ and $x=1)$ as the contribution from the Bessel function centred at the opposite corner will be non-zero along these lines. Nevertheless, the convergence using the Bessel functions is worse in this example.

In order to determine the reason for this relatively poor performance, the solution $J_{1}\left(\sqrt{\lambda} r^{\prime}\right) \sin \theta^{\prime}$ can be expanded in terms of Bessel functions centred on the origin. Using

$$
J_{n}\left(\sqrt{\lambda} r^{\prime}\right) e^{i n \theta^{\prime}}=\sum_{k=-\infty}^{\infty} J_{k-n}(\sqrt{\lambda} / 2) J_{k}(\sqrt{\lambda} r) e^{i k \theta},
$$

it follows that

$$
\begin{aligned}
J_{1}\left(\sqrt{\lambda} r^{\prime}\right) \sin \theta^{\prime} & =\sum_{k=1}^{\infty}\left(J_{k+1}(\sqrt{\lambda} / 2)+J_{k-1}(\sqrt{\lambda} / 2)\right) J_{k}(\sqrt{\lambda} r) \sin k \theta(15) \\
& =\frac{4}{\sqrt{\lambda}} \sum_{k=1}^{\infty} k J_{k}(\sqrt{\lambda} / 2) J_{k}(\sqrt{\lambda} r) \sin k \theta
\end{aligned}
$$

A similar expansion can be developed in terms of Bessel functions centred about $(1,0)$. The main difference between the series (16) and a series of the functions $B_{\alpha, k}$ is that the former includes all Bessel functions with order greater than 1, whereas the latter series has only the even order Bessel functions. According to (16) the Taylor expansion near the origin should contain all powers of $r$ greater than 1 , but the $B_{\alpha, k}$ contain only even powers. This suggests that an expansion in Bessel functions should contain the functions

$$
B_{\alpha, k}^{p}(r, \theta)=J_{\alpha+k}(\sqrt{\lambda} r) \sin (\alpha+k) \theta+J_{\alpha+k}\left(\sqrt{\lambda} r^{\prime \prime}\right) \sin (\alpha+k) \theta^{\prime \prime} .
$$

This expansion has been carried out and the result, shown in Figure 5, is a considerable improvement on the $B_{\alpha, k}$ expansion, but still not as good as the $W_{N, k}$ expansion. 

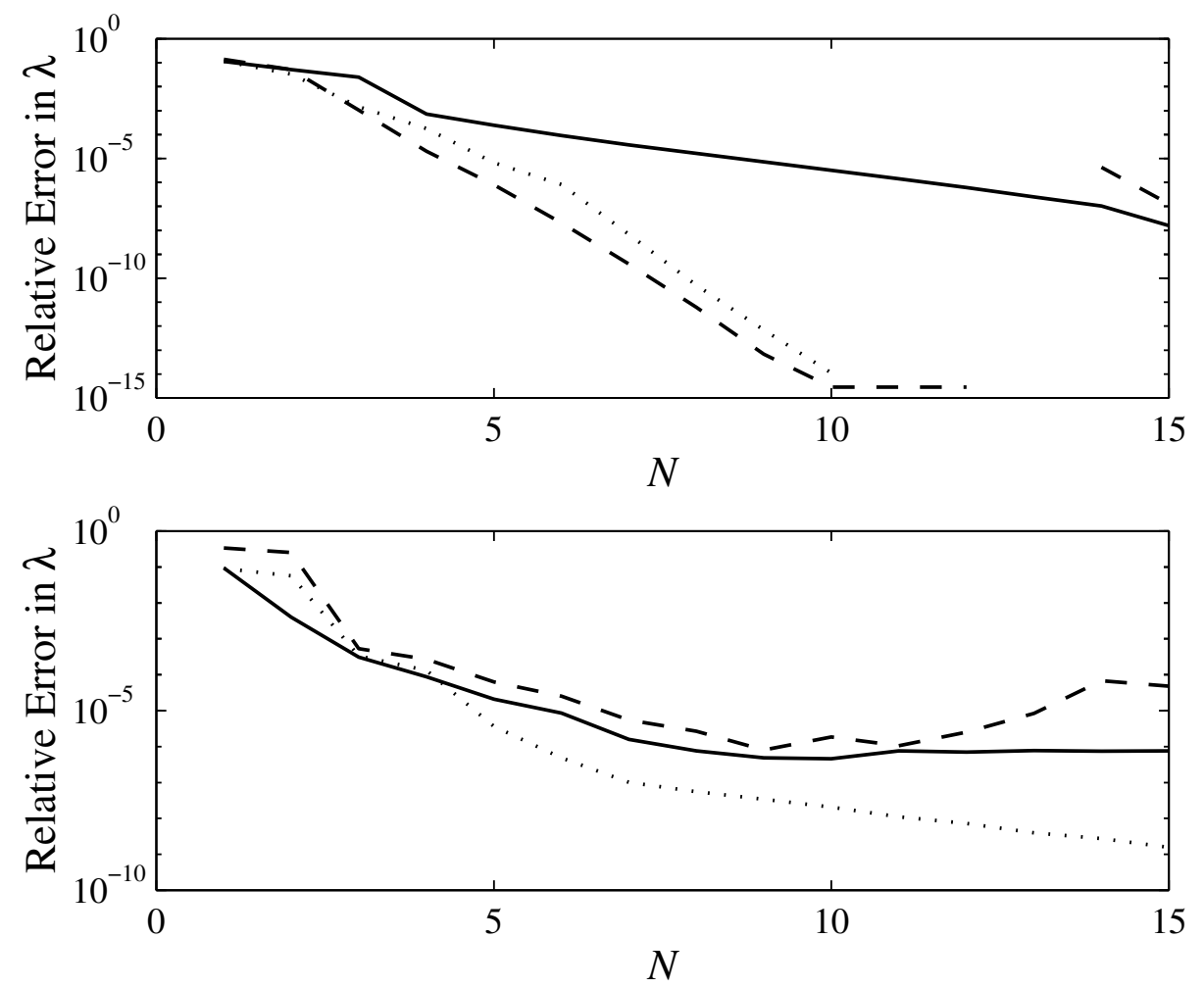

Figure 6: Convergence of the eigenvalue for $y=x(1-x)$ and $y=4 x(1-x)$ respectively for $W_{N, k}$ (dashed line), $B_{\alpha, k}$ (solid line) and $B_{\alpha, k}^{p}$ (dotted line). 


\subsection{Parabolic boundaries}

Lastly, the method is applied to regions with a parabolic upper boundary. The two cases chosen are the regions bounded by $y=x(1-x)$ and $y=$ $4 x(1-x)$. The latter corresponds to the curve $w=0$ in Figure 1 . The results are shown in Figure 6. In each of these cases, the solution was not known in advance, and so the eigenvalue had to be deduced from the numerical results. For the curve $y=x(1-x)$, expansions in $W_{N, k}$ and $B_{\alpha, k}^{p}$ both converged to the same value with almost machine accuracy. Again the expansion in $B_{\alpha, k}$ did not converge as rapidly, for reasons outlined above.

For the curve $y=4 x(1-x)$, expansions in $W_{N, k}$ were slow to converge. The likely explanation for this is that the corner at $(0,0)$ is singular in this case. This means that the solution will not be analytic at that point. On the other hand, the functions $W_{N, k}$ are themselves analytic everywhere, and so will not be able to match the solution accurately. The functions $B_{\alpha, k}$ perform marginally better because the first term, $B_{\alpha, 1}$ with $\alpha=\pi / \arctan (4)$, will match the first term, $r^{\alpha}$, in the series at $r=0$. However, the remaining functions in the expansion will not be able to match terms such as $r^{\alpha+1}$. The expansion in $B_{\alpha, k}^{p}$ performs significantly better than both of these. The most likely reason for this is that these functions are better able to match the powers of $r$ at the corners.

\section{Conclusion}

The modified method of particular solutions can give extremely accurate solutions for equations such as the Helmholtz equation. However, different choices of basis function lead to quite different convergence properties. We found that the functions $B_{\alpha, k}^{p}$ provided a better approximation than $B_{\alpha, k}$, at least where the sides adjacent to the corners were not straight line segments. Where the solution was analytic, the functions $W_{N, k}$ were also very good, 
but in cases where the solution was not analytic, they performed poorly.

In summary, it appears from these results that choosing the basis functions to match the boundary conditions near corners may not be as important as choosing functions that will be able to approximate the solution at those points where it is not analytic.

\section{References}

[1] L. Fox, P. Henrici and C. B. Moler, Approximations and bounds for eigenvalues of elliptic operators, SIAM J. Numer. Anal., 4:89-102, 1967. C545, C548

[2] W. W. Read, G. E. Sneddon and L. Bode, A series method for the eigenvalues of the advection diffusion equation, The ANZIAM Journal 45(E):C773-C786, 2004. C545, C546, C547

[3] T. Betcke and L. N. Trefethen, Reviving the method of particular solutions, SIAM Review, to appear. C545, C549

[4] B. J. McCartin, Eigenstructure of the equilateral triangle, SIAM Review, 45:267-287, 2003. C551 